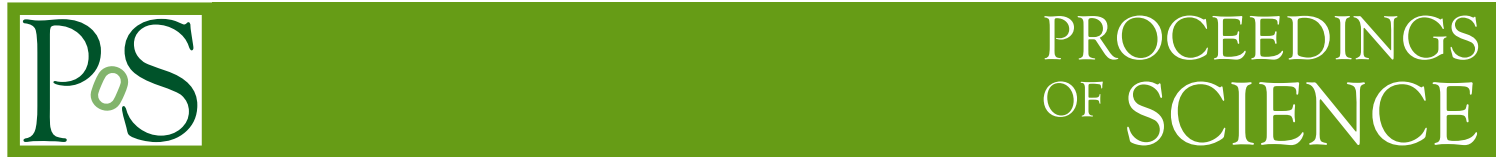

\title{
Beauty Production in Deep Inelastic Scattering at HERA using Decays into Electrons
}

\section{Ramoona Shehzadi*}

Physikalisches Institut der Universität Bonn

E-mail: shehzadi@physik.uni-bonn.de

The results from a recent analysis on beauty production in $e p$ interactions at HERA studied with the ZEUS detector for exchanged four-momentum squared $Q^{2}>10 \mathrm{GeV}^{2}$ are presented. The beauty events were identified using electrons from semileptonic $b$ decays with a transverse momentum $0.9<p_{T}^{e}<8 \mathrm{GeV}$ and pseudorapidity $\left|\eta^{e}\right|<1.5$. The fraction of events containing $b$ quarks was extracted from a likelihood fit using variables sensitive to electron identification as well as to semileptonic decays. Total and differential cross sections were measured and compared with next-to-leading-order QCD calculations. The beauty contribution to the proton structure function $F_{2}$ was extracted from the double-differential cross sections.

The 2011 Europhysics Conference on High Energy Physics, EPS-HEP 2011,

July 21-27, 2011

Grenoble, Rhône-Alpes, France

*on behalf of the ZEUS collaboration 


\section{Introduction}

The measurement of beauty production in $e p$ collisions at HERA provides a powerful tool for testing the proton structure and perturbative Quantum Chromodynamics ( $\mathrm{pQCD}$ ). The dominant production process is boson-gluon fusion between the incoming virtual photon and a gluon in the proton. Different kinematic variables which are used to describe ep interactions at HERA are: photon virtuality, $Q^{2}$, the Bjorken scaling variable, $x$, and the inelasticity, $y$. When $Q^{2}$ is large compared to the proton mass, the interaction is referred to as deep inelastic scattering (DIS).

In the analysis [1] presented here, beauty production was studied using semileptonic decays to electrons combined with lifetime information. The analysis was performed in the DIS regime $\left(Q^{2}>10 \mathrm{GeV}^{2}, 0.05<y<0.7\right)$. Electron candidates from semileptonic decays of $b$ quarks were required to have a transverse momentum $0.9<p_{T}^{e}<8 \mathrm{GeV}$ and pseudorapidity $\left|\eta^{e}\right|<1.5$. The measurements were compared to a leading order plus parton shower Monte Carlo (RAPGAP) [2] as well as QCD predictions at next-to-leading-order (NLO), calculated using HVQDIS program [3].

\section{Signal Extraction}

In oder to separate beauty signal from the background, discriminating variables sensitive to electron identification as well as to semileptonic decays were used. For the electron identification the measurement of the specific energy loss, $d E / d x$, in the central tracking detector; the ratio of the energy deposited in the calorimeter to the track momentum; and the penetrating depth of the energy deposited in the calorimeter were selected. Semileptonic decays were identified with the help of $p_{T}^{\text {rel }}$, the relative transverse momentum component of the electron candidate relative to the direction of jet axis; $\Delta \phi$, the difference of the azimuthal angle between the missing transverse momentum vector and the electron direction; and $d / \delta d$, the significance of the reconstructed decay length, where $d$ is defined as the distance in $X-Y$ between the secondary vertex and the interaction point, projected onto the jet axis. The six variables were combined into one discriminating test-function variable using a likelihood hypothesis. The distribution of likelihood test function is shown in Figure 1. The distribution was fit using the expected distributions of electrons from semileptonic $b$ decays, electrons from other sources and fake electrons, to determine the fractions of events from each source. The fit provides a very good description of data.

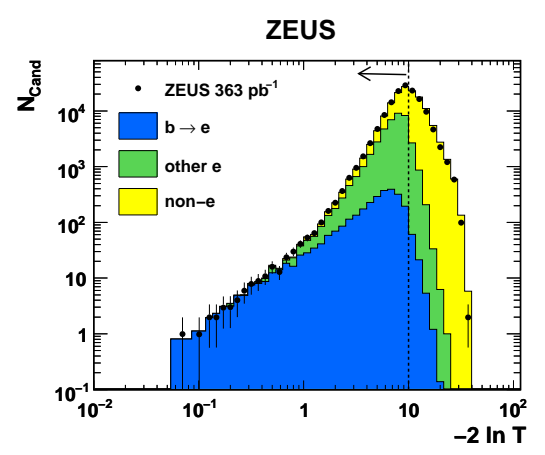

Figure 1: The distribution of $-2 \ln T$, where $\mathrm{T}$ is the likelihood test function.

\section{Results}

The total visible cross section and differential cross sections for $b$-quark production and the subsequent semileptonic decay to an electron with $p_{T}^{e}>0.9 \mathrm{GeV}$ in the range $\left|\eta^{e}\right|<1.5$ in DIS events with $Q^{2}>10 \mathrm{GeV}^{2}$ and $0.05<y<0.7$ were measured. Figure 2 shows differential cross sections as a function of $p_{T}^{e}$ and $\eta^{e}$ compared to the NLO QCD prediction and the RAPGAP MC scaled 


\section{ZEUS}

to the data. Both the descriptions from the NLO QCD calculation as well as the scaled RAPGAP cross sections describe the data well. The beauty contribution to the proton structure function $F_{2}$, denoted as $F_{2}^{b \bar{b}}$, was extracted from the double-differential cross section as a function of $Q^{2}$ and $x$. Figure 3 shows $F_{2}^{b \bar{b}}$ as a function of $Q^{2}$
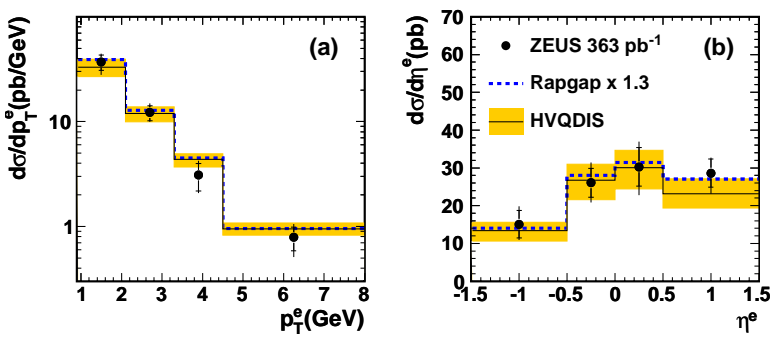

Figure 2: Differential cross sections for electrons from $b$ quark decays as a function of (a) $p_{T}^{e}$ and (b) $\eta^{e}$.

for fixed values of $x$. The results from this measurement have been compared with the previous measurements from the $\mathrm{H} 1$ and ZEUS collaborations. The different measurements are consistent with each other. Also the results are compared to several NLO and NNLO QCD predictions [4]. The data are reasonably well described by the different theory predictions.

\section{Summary}

A recent measurement of beauty production in DIS at HERA using decays into electrons was presented. A likelihood-ratio test function was used to identify the signal. The measured visible and differential cross sections are in agreement with the NLO QCD calculations. $F_{2}^{b \bar{b}}$ was extracted from the double differential cross sections and is in agreement with previous $\mathrm{H} 1$ and ZEUS measurements. For $Q^{2}>10 \mathrm{GeV}^{2}$, this measurement represents the most precise determination of $F_{2}^{b \bar{b}}$ by the ZEUS collaboration. The results were also compared to several NLO and NNLO QCD calculations, which provide a good description of the data.

\section{References}

[1] ZEUS Collaboration, H. Abramowicz et al., Eur. Phys. J. C 71, 1531 (2011).

[2] H. Jung, Comp. Phys. Comm. 86, 147 (1995).

[3] B.W. Harris and J. Smith, Phys. Rev. D 57, 28061998.

[4] P.M. Nadolsky et al., Phys. Rev. D 78, 013004 (2008);

A.D. Martin et al., Eur. Phys. J. C 63, 189 (2009);

R.S. Thorne, and W.K. Tung, Preprint arXiv:0809.0714, 2008;

M. Glück, P. Jimenez-Delgado and E. Reya, Eur. Phys. J. C 53, 355 (2008);

S. Alekhin and S. Moch, Phys. Lett. B 672, 166 (2009);

S. Alekhin et al., Phys. Rev. D 81, 014032 (2010). 